\title{
STUDY OF POSTURAL CONTROL AND BODY BALANCE IN SUBJECTS WITH DOWN SYNDROME
}

original paper

( ) University School of Physical Education in Wroclaw

DOI: https://doi.org/10.5114/hm.2021.98466

VALERIO GIUSTINO ${ }^{1,2}$, GIUSEPPE MESSINA ${ }^{2,3}$, MARIANNA ALESI ${ }^{2}$, LINDA LA MANTIA $^{3}$, ANTONIO PALMA $^{2,4,5}$, GIUSEPPE BATTAGLIA ${ }^{2,4,5}$

${ }^{1} \mathrm{PhD}$ Program in Health Promotion and Cognitive Sciences, University of Palermo, Palermo, Italy

${ }^{2}$ Department of Psychology, Educational Science and Human Movement, University of Palermo, Palermo, Italy

${ }^{3}$ Posturalab Italia Research Institute, Palermo, Italy

${ }^{4}$ Sport and Exercise Sciences Research Unit, University of Palermo, Palermo, Italy

${ }^{5}$ Regional Sports School of CONI Sicilia, Palermo, Italy

\begin{abstract}
Purpose. Body imbalance and poor postural control have negative impact on gait and may increase the risk of falling in healthy individuals as well as those with Down syndrome (DS). Since people with DS have lower physical levels than people who do not present this condition, the purpose of our research was to compare features of postural control and body balance between subjects affected by DS and a control sample.

Methods. Participants enrolled were divided into the following two groups: a DS group (DSG; $n=22$; 9 females and 13 males; age range: $16-36$ years) and an age-matched control group (CG; $n=25 ; 11$ females and 14 males; age range: $16-36$ years). All participants were subjected to two stabilometric tests: eyes open (EO) and eyes closed (EC), $30 \mathrm{~s}$ each, in bipedal upright stance on a platform.

Results. The main results showed significantly higher values for the ellipse sway area in DSG compared with CG, both in the $\mathrm{EO}(p=0.009)$ and in the $\mathrm{EC}$ trial $(p=0.000)$. Moreover, significantly higher values were revealed for the sway path length ( $p=0.013)$, maximum speed of sway $(p=0.001)$, and average speed of sway $(p=0.046)$ in the EC than in the EO trial.

Conclusions. We infer that a delay in multi-sensory integration processes in the nervous system for controlling body posture could underlie lower body balance in DS.
\end{abstract}

Key words: posture, postural control, body balance, stabilometric test, Down syndrome

\section{Introduction}

Down syndrome (DS), or trisomy 21 , is a genetic disorder characterized by intellectual disability, growth delay, and typical cranio-facial features. When compared with subjects with typical development (TD), subjects with this disorder often show a number of peculiar health issues, such as impairments in hearing and vision, as well as respiratory, masticatory, and swallowing dysfunctions [1, 2]. Recent research has demonstrated a relationship between motor competence and intellectual functioning. Indeed, DS subjects with mild or moderate intellectual disability exhibit motor competence delay with significant impairments in daily life activities reducing individual autonomy and influencing the social aspect [3, 4]. People affected by DS experience different effects in the cardiovascular and respiratory system in response to physical activity compared with TD individuals and, moreover, these subjects present unstable muscle activation patterns, poor postural control, motor incoordination, slow adaptation to environmental alterations, concurrent contraction of agonist and antagonist muscles, and dystonia [5-7]. From an early age, children with DS show impairments of postural control, body balance, and motor speed $[8,9]$. Authors have reported that children

Correspondence address: Valerio Giustino, PhD Program in Health Promotion and Cognitive Sciences, Department of Psychology, Educational Science and Human Movement, University of Palermo, Via Giovanni Pascoli, 6, 90144, Palermo, Italy, e-mail: valerio.giustino@unipa.it

Received: June 28, 2019

Accepted for publication: February 28, 2020

Citation: Giustino V, Messina G, Alesi M, la Mantia L, Palma A, Battaglia G. Study of postural control and body balance in subjects with Down syndrome. Hum Mov. 2021;22(1):66-71; doi: https://doi.org/10.5114/hm.2021.98466. 
with motor impairment lack acquired motor skills, have reduced gross motor performance, and exhibit a delay of social functioning [4, 8]. Muscle hypotonia and postural instability in children affected by DS both negatively affect gait patterns development $[10,11]$. In fact, muscle hypotonia appears to be strictly connected with the lower levels of postural control in DS subjects [12]. Body balance and postural control are important factors that impact on the skills for independence, with or without DS [13-16]. However, taskoriented postural control is consistently poor in DS and can influence basic physical activities such as running and jumping $[13,14,17]$.

A method for evaluating postural control and body balance is the study of the centre of pressure $(\mathrm{CoP})$ displacement. Specifically, the $\mathrm{CoP}$ is the point of application of the ground reaction force vector and it denotes the weighted average of all the pressure points of the feet surface in contact with the ground. The analysis of the CoP displacement in the time domain has been widely used to quantify postural control even in subjects with DS [18]. The CoP parameters include the ellipse sway area (ESA), i.e. the extent of the 2-dimensional figure containing 95\% of the CoP displacement; the sway path length (SPL), i.e. the linear length of the $\mathrm{CoP}$ displacement; the components of the $\mathrm{CoP}$ displacement on the frontal plane, i.e. right-left sway (X; X-mean), and on the sagittal plane, i.e. forward-backward sway (Y; Y-mean); the maximum speed of sway (Max-S); and the average speed of sway (AS). Several research groups have studied postural control in TD subjects as well as in those with DS [11, 18]. Hence, the purpose of this study was to evaluate any difference in postural control and body balance in individuals with and without DS. We hypothesize that the DS group (DSG) may show impaired postural abilities (i.e. higher variability in the CoP parameters) when compared with the control group (CG).

\section{Material and methods}

\section{Participants}

In this cross-sectional study we recruited 50 participants, 25 with DS (12 females and 13 males; age range: $16-36$ years) and 25 age-matched control subjects with TD (11 females and 14 males; age range: $16-36$ years). After being interviewed, 22 of the 25 participants with trisomy 21 (9 females and 13 males) respected all inclusion criteria and were enrolled in our study. DS subjects were included in the study if they met the inclusion criteria as follows: the presence of trisomy 21; moderate level of intellectual disability (certified by the National Public Health Institute); no physical condition limiting the capacity to stand unassisted and no gait impairment. DS participants with translocations or severe intellectual disability were excluded from the study $(n=3)$. For TD individuals, the inclusion criteria were simply the absence of any physical condition limiting the capacity to stand unassisted or any gait impairment. Hence, the participants were divided into the following two groups: DSG and the age-matched CG, as reported in Table 1.

Subjects with DS were recruited through a local hospital (see the Acknowledgments section) and a local not-for-profit association that operates for people with DS and supports their families (see the Acknowledgments section). The TD participants consisted of students from Palermo (secondary school and university). A full clinical history was collected for each participant, including pathological conditions, prior surgical procedures, and hospital admissions. All individuals were administered a questionnaire so as to collect personal and health-status data.

\section{Anthropometric assessment}

Body weight $(\mathrm{kg})$ and height $(\mathrm{m})$ were collected in accordance with the standardized procedures drawn up at the Airlie Conference [19]. Body mass index (BMI) was determined by multiplying the body weight by the height squared $\left(\mathrm{kg} / \mathrm{m}^{2}\right)$.

\section{Body posture assessment}

Posturographic measurement was carried out in a silent room to avoid sources of external distraction. The participants were instructed to stand barefoot and upright for $30 \mathrm{~s}$ on a stabilometric platform, with their arms at their sides and feet forming a $30^{\circ}$ angle with heels $4 \mathrm{~cm}$ apart [20, 21]. The stabilometric testing was conducted firstly with eyes open (EO) and then repeated with eyes closed (EC). In the EO condition, the subjects were required to gaze at an eye-level point at 1-m distance. So as to familiarize the participants with the procedure and the laboratory setting, a trial run, prior to testing, was allowed. Standardized verbal instructions were given to each individual. The stabilometric test was conducted with the freeMed ${ }^{\circledR}$ platform and the freeStep ${ }^{\circledast}$ software produced by Sensor Medica ${ }^{\circledR}$ (Guidonia Montecelio, Italy). The acquired data were transformed into $\mathrm{CoP}$ coordinates by the software. The following parameters were selected for both EO and EC conditions: ESA, SPL, CoP coordinates 
along the frontal (X; X-mean) and sagittal (Y; Y-mean) planes, Max-S, minimum speed of sway (Min-S), and AS.

\section{Statistical analysis}

Data presented as mean values \pm standard deviations were analysed by using the Statistica 12 software (StatSoft $^{\oplus}$, TIBCO $^{\circledR}$ Software Inc., Palo Alto, USA). Differences between groups were calculated via unpaired-sample $t$-tests, whereas within-group comparisons under $\mathrm{EO}$ and $\mathrm{EC}$ conditions were computed via paired-sample $t$-tests. The level of alpha $<0.05$ was considered significant for all analyses.

\section{Ethical approval}

The research related to human use has complied with all the relevant national regulations and institutional policies, has followed the tenets of the Declaration of Helsinki, and has been approved by the Ethical Board of the University of Palermo.

\section{Informed consent}

Informed consent has been obtained from all individuals included in this study or their legal guardians.

\section{Results}

At recruitment, no significant differences $(p>0.05)$ were found between DSG and CG regarding gender distribution or age mean, as shown in Table 1. However, in accordance with the reported literature, the unpaired $t$-test analysis showed significant differences in weight, height, and BMI (Table 1).

With regard to the postural features with EO, DSG presented significantly higher values in Max-S ( $p=$ $0.003)$, Min-S $(p=0.036)$, and AS $(p=0.029)$ compared with CG. During postural analysis with EC, DSG showed significant differences in comparison with CG in Max-S $(p=0.003)$ and AS ( $p=0.005)$ (Table 2).

Table 1. Characteristics of the sample

\begin{tabular}{lccc}
\hline Parameter & DSG $(n=22)$ & CG $(n=25)$ & $p$ \\
\hline Gender M/F (\%) & $13 / 9(59 / 41)$ & $14 / 11(56 / 44)$ & n.s. \\
Age (years) & $25.72 \pm 4.83$ & $27.4 \pm 3.24$ & n.s. \\
Height $(\mathrm{m})$ & $1.49 \pm 0.08$ & $1.64 \pm 0.09$ & $<0.001$ \\
Weight $(\mathrm{kg})$ & $64.18 \pm 11.15$ & $57.04 \pm 10.86$ & 0.031 \\
BMI $\left(\mathrm{kg} / \mathrm{m}^{2}\right)$ & $28.94 \pm 4.9$ & $21.11 \pm 2.34$ & $<0.001$ \\
\hline
\end{tabular}

DSG - Down syndrome group, CG - control group,

$\mathrm{M}$ - male, F - female, BMI - body mass index, n.s. - not significant (the significance level adopted was $p<0.05$ )
Table 2. Study of postural parameters in the sample

\begin{tabular}{|c|c|c|c|}
\hline \multirow{2}{*}{ Parameter } & \multicolumn{3}{|c|}{$\mathrm{EO}$} \\
\hline & $\operatorname{DSG}(n=22)$ & $\mathrm{CG}(n=25)$ & $p$ \\
\hline $\operatorname{ESA}\left(\mathrm{mm}^{2}\right)$ & $108.17 \pm 102.89$ & $43.33 \pm 55.91$ & 0.009 \\
\hline SPL (mm) & $675.08 \pm 222.75^{\mathrm{a}}$ & $828.03 \pm 349.75$ & n.s. \\
\hline $\operatorname{Max}-\mathrm{S}(\mathrm{mm} / \mathrm{s})$ & $3.44 \pm 1.71^{\mathrm{a}}$ & $2.25 \pm 0.88^{b}$ & 0.003 \\
\hline Min-S (mm/s) & $0.03 \pm 0.02$ & $0.01 \pm 0.02$ & 0.036 \\
\hline $\mathrm{AS}(\mathrm{mm} / \mathrm{s})$ & $20.89 \pm 7.2^{\mathrm{a}}$ & $16.31 \pm 6.81$ & 0.029 \\
\hline $\mathrm{X}(\mathrm{mm} / \mathrm{s})$ & $-5.22 \pm 9.29$ & $-4.31 \pm 6.06$ & n.s. \\
\hline $\mathrm{Y}(\mathrm{mm} / \mathrm{s})$ & $-9.47 \pm 10.84$ & $-14.24 \pm 8.43$ & n.s. \\
\hline \multirow{2}{*}{ Parameter } & \multicolumn{3}{|c|}{$\mathrm{EC}$} \\
\hline & $\operatorname{DSG}(n=22)$ & CG $(n=25)$ & $p$ \\
\hline $\operatorname{ESA}\left(\mathrm{mm}^{2}\right)$ & $137.12 \pm 126.94$ & $41.04 \pm 41.77$ & $<0.001$ \\
\hline SPL (mm) & $863.04 \pm 293.48^{\mathrm{a}}$ & $845.8 \pm 329.56$ & n.s. \\
\hline $\operatorname{Max}-\mathrm{S}(\mathrm{mm} / \mathrm{s})$ & $10.62 \pm 8.9^{a}$ & $4.54 \pm 4.22^{\mathrm{b}}$ & 0.003 \\
\hline Min-S (mm/s) & $0.02 \pm 0.02$ & $0.02 \pm 0.02$ & n.s. \\
\hline $\mathrm{AS}(\mathrm{mm} / \mathrm{s})$ & $25.7 \pm 8.33^{\mathrm{a}}$ & $17.95 \pm 9.53$ & 0.005 \\
\hline $\mathrm{X}(\mathrm{mm} / \mathrm{s})$ & $-8.36 \pm 14.92$ & $-4.99 \pm 5.66$ & n.s. \\
\hline $\mathrm{Y}(\mathrm{mm} / \mathrm{s})$ & $-7.81 \pm 8.67$ & $-12.82 \pm 8.99$ & n.s. \\
\hline
\end{tabular}

EO - eyes open, EC - eyes closed, DSG - Down syndrome group, CG - control group, ESA - ellipse sway area, SPL - sway path length, Max-S - maximum speed of sway, Min-S - minimum speed of sway, AS - average speed of sway,

X (right-left) - X-mean, Y (forward-backward) - Y-mean, n.s. - not significant (the significance level adopted was $p<0.05)$

${ }^{a} p<0.05$, DSG with EO vs. DSG with EC ${ }^{\mathrm{b}} p<0.05$, CG with EO vs. CG with EC

As can be observed in Figure 1, we found a significantly higher ESA in DSG compared with CG during postural analysis in the EO $(p=0.009)$ and EC ( $p=$ 0.000 ) conditions. Moreover, the paired $t$-test analyses between the EO and EC trials in DSG revealed significant differences in SPL ( $p=0.013)$, Max-S ( $p=0.001)$, AS ( $p=0.046)$, whereas CG showed only a significantly higher Max-S $(p=0.01)$.

\section{Discussion}

The purpose of this study was to investigate postural control and body balance in subjects affected by DS as compared with controls with TD. In line with several studies in the literature, people with DS showed significant anthropometric differences in comparison with individuals with TD $(p<0.05)$ [22, 23]. Indeed, Zemel et al. [22] reported that, on average, subjects with DS had a significantly lower stature, as well as higher weight and BMI in comparison with subjects with TD. The authors described the patients' short 


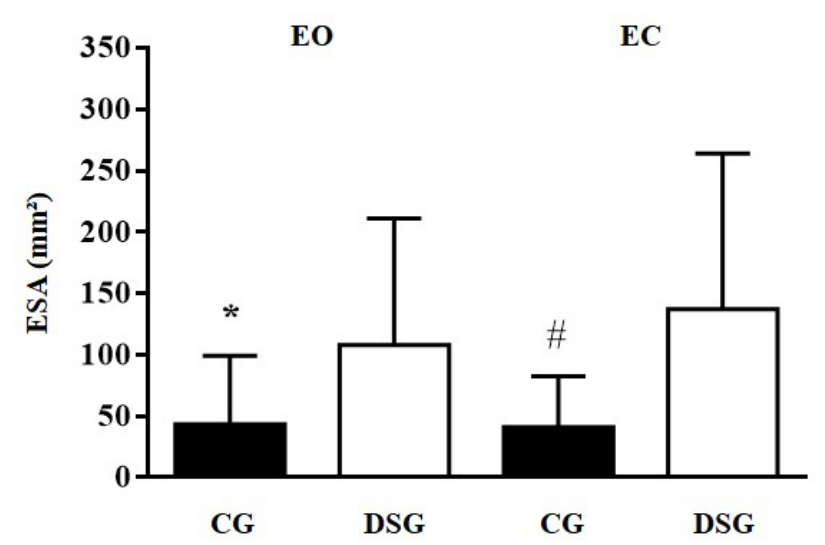

${ }^{*} p<0.01, \# p<0.001$

Figure 1. Study of the ellipse sway area (ESA) parameter during postural analysis with eyes open (EO) and eyes closed (EC) in the Down syndrome group (DSG) and control group (CG)

stature, small head circumference, and normal to high relative body weight, typical of the genetic syndrome [22]. In our study, postural analysis was performed during an upright position with EO and EC. Even though the capacity to maintain an orthostatic bipedal position may seem very easy, it necessitates the integration of afferences to the central nervous system from the vestibular apparatus, proprioceptive organs, as well as visual and auditory pathways [24, 25]. During postural assessment with EO, we observed higher ESA ( $p=$ $0.009)$, Max-S ( $p=0.003)$, Min-S $(p=0.036)$, and AS $(p=0.029)$ in individuals affected by DS compared with CG. These data are in line with the outcomes achieved by Cimolin et al. [26], who observed a reduced capacity in subjects with DS to preserve CoP into their base of support when compared with individuals with TD [26]. This is likely due to the fact that DS entails several cognitive and motor deficits. Indeed, it is wellknown that DS-related cognitive impairment may result in reduced integration processes in the nervous system, slower decision making, lower ability to integrate multi-sensory information, longer simple reaction times [27], reduced grip strength [28], and worse anticipatory postural adjustments [10]. DS-related muscle weakness and hypotonia, which affect hip and ankle strategies of postural control, are potentially destabilizing. Moreover, muscle reaction velocities are significantly slower in DS, resulting in greater difficulty in motor control and agility [10, 11, 18, 27]. All of these impairments may negatively influence the development of the postural control system, which is less able to adjust and adequately respond to environmental requirements. As for the postural assessment with EC, our findings showed significantly higher values of ESA ( $p=0.000), \operatorname{Max}-\mathrm{S}(p=0.003)$, and AS ( $p=$ $0.005)$ in DSG compared with CG. The optimal condition for maintaining an upright posture is when there is no interference of sensory input and, thus, less involvement of the postural control system. In a number of studies, an increase in body sway has been observed when one or more input of the multi-sensory stimuli were excluded or otherwise altered experimentally, which required more active participation on the part of the postural control system [18, 29]. Moreover, our outcomes reported significantly higher values of SPL ( $p=0.013)$, Max-S $(p=0.001)$, and AS $(p=0.046)$ in DSG during the stabilometric trial with EC compared with EO. In accordance with the literature, our results showed a lower level of postural control during the analysis with EC than that with EO [30]. Vision is particularly important for maintaining postural control and body balance. As with TD subjects, those with DS maintain their balance with greater difficulty with EC than with EO. This underlines that postural instability is higher with EC and improves in the presence of visual feedback, thus highlighting the primary importance of vision for body balance and postural control [30].

\section{Conclusions}

In DS individuals, postural control should be improved in order to increase the overall function and prevent the risk of falling. Hence, it is important to promote the practice of physical activity among people with DS and to encourage them to perform adapted training programs, including exercises aimed at improving body balance, thus reinforcing their capacity to interact with their surroundings with less fear of falling [31]. These adapted training programs should focus on somatosensory input and proprioceptive exercises, but also seek to potentiate strength and coordination, ultimately responsible for coordinated musculoskeletal responses [12, 32]. The presented data could be used as a groundwork for developing specific training programs, as well as to set physical treatment goals in order to improve the activities of daily life in people with intellectual disability. However, our results need to be interpreted with caution, given the small sample size.

\section{Acknowledgments}

The authors would like to thank all subjects that were involved in the study and their parents. Moreover, we would like to thank Dr. Maria Piccione and the local hospital participating in the study (i.e. Azienda Ospedali Riuniti Villa Sofia-Cervello, Palermo, Italy) 
and Giampiero Gliubizzi and the local not-for-profit associations (i.e. A.S.D. Sport21 Sicilia, Palermo, Italy; Associazione Famiglie Persone Down, Palermo, Italy) for the collaboration during the study. Finally, we thank John De Simone for his contribution regarding the English revision of the final manuscript.

\section{Disclosure statement}

No author has any financial interest or received any financial benefit from this research.

\section{Conflict of interest}

The authors state no conflict of interest.

\section{References}

1. Roizen NJ, Magyar CI, Kuschner ES, Sulkes SB, Druschel C, van Wijngaarden E, et al. A community crosssectional survey of medical problems in 440 children with Down syndrome in New York State. J Pediatr. 2014;164(4):871-875; doi:10.1016/j.jpeds.2013.11.032.

2. Hashimoto M, Igari K, Hanawa S, Ito A, Takahashi A, Ishida N, et al. Tongue pressure during swallowing in adults with Down syndrome and its relationship with palatal morphology. Dysphagia. 2014;29(4):509-518; doi: 10.1007/s00455-014-9538-5.

3. Ferreira-Vasques AT, Cusin Lamônica DA. Motor, linguistic, personal and social aspects of children with Down syndrome. J Appl Oral Sci. 2015;23(4):424-430; doi: 10.1590/1678-775720150102.

4. Malak R, Kostiukow A, Krawczyk-Wasielewska A, Mojs E, Samborski W. Delays in motor development in children with Down syndrome. Med Sci Monit. 2015;21:1904-1910; doi: 10.12659/MSM.893377.

5. Da Silva Bezerra ME, de Deus LA, dos Santos Rosa T, da Silva EEL, Simões HG, Vieira E. Acute effects of cycling exercise on post-exercise blood pressure in individuals with Down syndrome. Hum Mov. 2017; 18(4):61-66; doi: 10.1515/humo-2017-0036.

6. Fernhall B, Pitetti KH, Rimmer JH, McCubbin JA, Rintala P, Millar AL, et al. Cardiorespiratory capacity of individuals with mental retardation including Down syndrome. Med Sci Sports Exerc. 1996;28(3):366-371; doi: 10.1097/00005768-199603000-00012.

7. Gontijo APB, Mancini MC, Silva PLP, Chagas PSC, Sampaio RF, Luz RE, et al. Changes in lower limb cocontraction and stiffness by toddlers with Down syndrome and toddlers with typical development during the acquisition of independent gait. Hum Mov Sci. 2008; 27(4):610-621; doi: 10.1016/j.humov.2008.01.003.

8. Das Neves Cardoso AC, de Campos AC, dos Santos MM, Cabrera Santos DC, Ferreira Rocha NAC. Motor performance of children with Down syndrome and typical development at 2 to 4 and 26 months. Pediatr Phys Ther. 2015;27(2):135-141; doi: 10.1097/PEP.0000000 000000120 .
9. Malak R, Kotwicka M, Krawczyk-Wasielewska A, Mojs E, Samborski W. Motor skills, cognitive development and balance functions of children with Down syndrome. Ann Agric Environ Med. 2013;20(4):803-806.

10. Shumway-Cook A, Woollacott MH. Dynamics of postural control in the child with Down syndrome. Phys Ther. 1985;65(9):1315-1322; doi: 10.1093/ptj/65.9.1315.

11. Rigoldi C, Galli M, Mainardi L, Crivellini M, Albertini G. Postural control in children, teenagers and adults with Down syndrome. Res Dev Disabil. 2011;32(1): 170-175; doi: 10.1016/j.ridd.2010.09.007.

12. Davis WE, Kelso JA. Analysis of "invariant characteristics” in the motor control of Down's syndrome and normal subjects. J Mot Behav. 1982;14(3):194-212; doi: 10.1080/00222895.1982.10735273.

13. Wang H-Y, Long I-M, Liu M-F. Relationships between task-oriented postural control and motor ability in children and adolescents with Down syndrome. Res Dev Disabil. 2012;33(6):1792-1798; doi: 10.1016/j. ridd.2012.05.002.

14. Wang W-Y, Ju Y-H. Promoting balance and jumping skills in children with Down syndrome. Percept Mot Skills. 2002;94(2):443-448; doi: 10.2466/pms.2002. 94.2.443.

15. Battaglia G, Bellafiore M, Bianco A, Paoli A, Palma A. Effects of a dynamic balance training protocol on podalic support in older women. Pilot study. Aging Clin Exp Res. 2010;22(5-6):406-411; doi: 10.1007/BF033 37736.

16. Bellafiore M, Battaglia G, Bianco A, Paoli A, Farina F, Palma A. Improved postural control after dynamic balance training in older overweight women. Aging Clin Exp Res. 2011;23(5-6):378-385; doi: 10.1007/BF033 37762.

17. Ulrich DA, Ulrich BD, Angulo-Kinzler RM, Yun J. Treadmill training of infants with Down syndrome: evidence-based developmental outcomes. Pediatrics. 2001;108(5):E84; doi: 10.1542/peds.108.5.e84.

18. Galli M, Rigoldi C, Mainardi L, Tenore N, Onorati P, Albertini G. Postural control in patients with Down syndrome. Disabil Rehabil. 2008;30(17):1274-1278; doi: 10.1080/09638280701610353.

19. Lohman TG, Roche AF, Martorell R (eds.). Anthropometric standardization reference manual. Champaign: Human Kinetics; 1988.

20. Duarte M, Freitas SMSF. Revision of posturography based on force plate for balance evaluation. Rev Bras Fisioter. 2010;14(3):183-192; doi: 10.1590/S141335552010000300003.

21. Scoppa F, Gallamini M, Belloni G, Messina G. Clinical stabilometry standardization: feet position in the static stabilometric assessment of postural stability. Acta Med Mediterr. 2017;33:707-713; doi: 10.19193/03936384_2017_4_105.

22. Zemel BS, Pipan M, Stallings VA, Hall W, Schadt K, Freedman DS, et al. Growth charts for children with 
Down syndrome in the United States. Pediatrics. 2015; 136(5):e1204-e1211; doi: 10.1542/peds.2015-1652.

23. Asha KR, Lakshmiprabha S, Nanjaiah CM, Prashanth SN. Craniofacial anthropometric analysis in Down syndrome. Indian J Pediatr. 2011;78(9):1091-1095; doi: 10.1007/s12098-011-0377-1.

24. Gomes MM, Barela JA. Postural control in down syndrome: the use of somatosensory and visual information to attenuate body sway. Motor Control. 2007;11(3):224234; doi: 10.1123/mcj.11.3.224.

25. Thomas E, Martines F, Bianco A, Messina G, Giustino V, Zangla D, et al. Decreased postural control in people with moderate hearing loss. Medicine. 2018;97(14): e0244; doi: 10.1097/md.0000000000010244.

26. Cimolin V, Galli M, Vismara L, Grugni G, Priano L, Capodaglio $\mathrm{P}$. The effect of vision on postural strategies in Prader-Willi patients. Res Dev Disabil. 2011; 32(5):1965-1969; doi: 10.1016/j.ridd.2011.04.002.

27. Latash ML. The First Conference on Motor Control in Down Syndrome. J Mot Behav. 1990;22(3):444-446; doi: 10.1080/00222895.1990.10735522.

28. Cole KJ, Abbs JH, Turner GS. Deficits in the production of grip forces in Down syndrome. Dev Med Child Neurol.1988;30(6):752-758; doi:10.1111/j.1469-8749. 1988.tb14637.x..

29. Meneghetti CHZ, Blascovi-Assis SM, Deloroso FT, Rodrigues GM. Static balance assessment among children and adolescents with Down syndrome. Braz J Phys Ther. 2009;13(3):230-235; doi: 10.1590/S1413-355520090 05000029 .

30. Collins JJ, De Luca CJ. The effects of visual input on open-loop and closed-loop postural control mechanisms. Exp Brain Res. 1995;103(1):151-163; doi: 10.1007/ BF00241972.

31. Jankowicz-Szymanska A, Mikolajczyk E, Wojtanows$\mathrm{ki}$ W. The effect of physical training on static balance in young people with intellectual disability. Res Dev Disabil. 2012;33(2):675-681; doi: 10.1016/j.ridd.2011. 11.015 .

32. Alesi M, Battaglia G, Roccella M, Testa D, Palma A, Pepi A. Improvement of gross motor and cognitive abilities by an exercise training program: three case reports. Neuropsychiatr Dis Treat. 2014;10:479-485; doi: 10.2147/ndt.s58455. 